\title{
La familia Apocynaceae sensu lato en México: diversidad y distribución
}

\author{
The family Apocynaceae sensu lato in Mexico: diversity and distribution
}

\author{
Verónica Juárez-Jaimes*, Leonardo O. Alvarado-Cárdenas y José Luis Villaseñor \\ Departamento de Botánica, Instituto de Biología, Universidad Nacional Autónoma de México, Apartado postal 70-233, 04510, México, D. F. \\ *Correspondencia: vjuarez@ibiologia.unam.mx
}

\begin{abstract}
Resumen. La familia Apocynaceae s.l. se ubica entre las 15 familias más diversas de México, con 385 especies repartidas en 50 géneros y 3 subfamilias, de las cuales Asclepiadoideae es la de mayor diversidad en géneros, especies y endemismos. Los géneros con más especies nativas (y endémicas) son Asclepias y Matelea. Los estados más ricos en diversidad de taxones son los ubicados en ambas vertientes; destacan Chiapas, Guerrero, Oaxaca y Veracruz. Por su número de especies, sobresalen el bosque tropical caducifolio y los matorrales xerófilos. Del total de especies, 176 son endémicas de México. Se presenta la lista de especies registradas, señalándose su distribución por estados.
\end{abstract}

Palabras clave: Apocynaceae s.l., Apocynoideae, Asclepiadoideae, Rauvolfioideae, Diversidad.

\begin{abstract}
Apocynaceae s.l. is among the 15 most diverse families in Mexico, with a total of 385 species, distributed in 50 genera and 3 subfamilies of which Asclepiadoideae is the most diverse in numbers of genera, species and endemics. The genera with the most native species and endemics are Asclepias and Matelea. The richest states in terms of taxon diversity are those at the Pacific and Atlantic slopes, Chiapas, Oaxaca, Guerrero and Veracruz being the most prominent. Tropical deciduous forest and arid tropical scrub are the most important vegetation types by their total number of species, 176 of which are endemic to Mexico. A list of species recorded in México is provided, indicating their state distribution.
\end{abstract}

Key words: Apocynaceae s.l., Apocynoideae, Asclepiadoideae, Rauvolfioideae, species diversity.

\section{Introducción}

Jussieu (1798) propuso por primera vezlas Apocynaceae bajo una circunscripción poco clara, reconociendo 3 grupos con base en las características de los frutos. En 1810, Robert Brown separó las Asclepiadaceae de la circunscripción de Jussieu, apoyado en su compleja morfología floral. Desde entonces se ha aceptado tal propuesta y se han reconocido como 2 familias independientes, aunque cercanamente relacionadas.

En fechas recientes, la información morfológica y sobre todo los análisis filogenéticos basados en datos moleculares (Sennblad y Bremer, 1996, 2002) y combinados (Endress et al., 1996; Potgieter y Albert, 2001) han aportado suficiente evidencia para reconsiderar la unificación de las 2 familias en una sola (Apocynaceae sensu lato). Con el fin de reconocer sólo grupos monofiléticos, esta decisión parece recomendable, ya que de otro modo se continuaría manteniendo una propuesta de arreglo artificial. De esta manera, las Apocynaceae s.l. se siguen reconociendo como

Recibido: 14 noviembre 2006; aceptado: 27 febrero 2007 parte del orden Gentianales y actualmente se subdividen en 5 subfamilias de acuerdo con Endress y Bruyns (2000): Apocynoideae Burnett, Asclepiadoideae R. Br. ex Burnett, Periplocoideae R. Br. ex Endl., Rauvolfioideae Kostel y Secamonoideae Endl. que incluyen alrededor de 395 géneros y 5000 especies (Endress, 2004), y tanto en el mundo como en México se ubican entre las familias más ricas por su número de especies.

La nueva propuesta de clasificación es un paso importante hacia un mejor conocimiento de su historia natural y en particular de su sistemática, como lo evidencian los trabajos de Liede y Täuber (2000), Rapini et al. (2003) o Liede-Schumann et al. (2005). Desafortunadamente, en términos geográficos, tal conocimiento no es equitativo; por ejemplo, las investigaciones llevadas a cabo en el Viejo Mundo han generado bastante información, lo que no ha ocurrido en América. De manera particular, en México persiste la tradición de considerar Apocynaceae y Asclepiadaceae como familias distintas (Standley, 1924; Williams, 1996b; Juárez-Jaimes y Lozada, 2003; AlvaradoCárdenas, 2004a). El objetivo de este trabajo es presentar un resumen sobre el conocimiento de la diversidad y 
distribución geográfica de Apocynaceae s.l. en México.

\section{La familia Apocynaceae s.l.}

Aunque ya existen trabajos que tratan Apocynaceae y Asclepiadaceae como una sola entidad taxonómica, en este trabajo se presenta un resumen de las principales características de los taxones mexicanos, considerando conveniente proporcionar una descripción del grupo. Se discuten también algunos aspectos de su potencial importancia antropocéntrica.

La familia Apocynaceae incluye plantas anuales o perennes, principalmente hierbas erectas o trepadoras y con menos frecuencia árboles y arbustos. La mayoría de sus integrantes están provistos de laticíferos constituidos por células individuales o ramificadas que producen látex lechoso, rojizo (Aspidosperma) o transparente (Thenardia), el cual contiene glucósidos y alcaloides que pueden ser muy tóxicos (Asclepias linaria Cav.). Las hojas son simples, persistentes o caducas, opuestas o a veces alternas, rara vez verticiladas, acompañadas por glándulas conocidas como coléteres; los coléteres pueden localizarse en las axilas, en la base y sobre el pecíolo o en la haz a lo largo del nervio medio, o incluso en las bases de las brácteas y de los sépalos. En la familia se presentan tanto flores solitarias, surgiendo de la parte terminal de las ramas o en las axilas de las hojas (Mandevilla), como una variedad muy amplia de inflorescencias, sobre todo racemosas; por ejemplo, umbelas (Asclepias), corimbos (Cascabela), racimos (Mandevilla) o panículas. Las flores son hermafroditas, actinomorfas o ligeramente zigomorfas, pentámeras, vistosas o muy pequeñas (Orthosia, Metastelma, Rauvolfia); el cáliz es gamosépalo, pero dividido casi hasta su base, la corola es gamopétala, hipocraterimorfa, infudibuliforme o con menos frecuencia tubular o urceolada; el limbo presenta una prefloración dextrocontorta o sinistrocontorta.

En la corola de muchas de las especies se presentan estructuras que dependiendo de la subfamilia se denominan apéndices supraestaminales, apéndices infraestaminales, corona o corona corolina, de posición y forma variable (e.g. anulares [Prestonia, Laubertia], estaminoidales [Cascabela], cojinetes [Marsdenia], etc.).

Las anteras son biloculares o tetraloculares, basifijas; pueden estar libres, aglutinadas o fusionadas a la cabeza estigmática. El polen está agregado en mónadas, tétradas o aglutinado en una masa cerosa por lóculo llamado polinio. Los polinios de estambres adyacentes se unen a los brazos flexibles de un translator, unidos, a su vez, a un corpúsculo, el cual se ubica entre las anteras. El ovario es súpero (en las especies de Plumeria, semiínfero), principalmente apocárpico, rara vez sincárpico; los óvulos son numerosos, dispuesto en una placentación marginal o axilar. La parte superior del gineceo constituye una estructura especializada, de forma variable denominada estigma, cabeza estilar o cabeza estigmática, que puede ser receptiva a todo lo largo o solamente en su posición basal.

Enlafamilia se muestraunincrementoen la organización del androceo con la cabeza estigmática, hasta formar el ginostegio. Por ejemplo, los miembros de la subfamilia Rauvolfioideae presentan anteras no especializadas y libres de la cabeza estigmática, con excepción de algunos géneros, como Cascabela, Stemmadenia y Tabernaemontana; en las otras subfamilias las anteras se unen al estigma formando el ginostegio. En la subfamilia Apocynoideae las anteras son especializadas con rieles guías lignificados, pero sin translatores. Las subfamilias Periplocoideae, Secamonoideae y Asclepiadoideae tienen translatores. Periplocoideae se caracteriza por las anteras sin rieles guías lignificados y por el polen en tétradas libres o agregado en polinios en un translator, con un receptáculo en forma de cuchara. Secamonoideae tiene los granos de polen agregados en 4 diminutos polinios, unidos directa o indirectamente al corpúsculo. Asclepiadoideae presenta estructuras muy especializadas, donde las anteras se unen a la cabeza estigmática formando el ginostegio, el tubo estaminal generalmente está asociado a una corona dorsal y con rieles guía muy esclerificados y los granos de polen siempre están contenidos en polinios que cuentan con translatores.

Los frutos son muy variados; generalmente son folículos (Gonolobus, Mandevilla, Marsdenia, Plumeria), pero también se presentan drupas (Cascabela), bayas (Vallesia) y, rara vez, sámaras (Cameraria). Las semillas son aplanadas y comosas, ciliadas, aladas, cubiertas por un arilo (Stemmadenia y Tabernaemontana) o desnudas.

Importancia antropogénica de Apocynaceae s.l.

Desde mucho tiempo atrás, algunas especies de la familia Apocynaceae s.l. se han utilizado como veneno (Woodson, 1930); otras se utilizan en la actualidad como plantas de ornato o fármaco efectivo contra la leucemia (Catharanthus roseus (L.) G. Don). Varias especies provenientes del Viejo Mundo son muy apreciadas en México como ornamentales (por ejemplo, Nerium oleander L., Vinca major L. o Ceropegia woodii Schltr.), en tanto que Matelea cyclophylla (Standl.) Woodson, endémica de México, se cultiva en el Viejo Mundo junto con otras especies americanas, como Allamanda cathartica L., Cascabela thevetia (L.) Lippold o Plumeria rubra L. En la región mesoamericana, algunas especies de Plumeria tienen importancia ceremonial. El "hueso" (endocarpo pétreo) de las especies de Cascabela se emplea en México como instrumento musical por grupos que ejecutan danzas 
de intención prehispánica.

Aun cuando la familia está integrada por plantas tóxicas, algunas han sido incluidas como condimento de platillos regionales; por ejemplo, en varias zonas de la costa del Pacífico (Guerrero, Oaxaca, Sonora), los frutos verdes de Marsdenia y Gonolobus se consumen asados; en la depresión central de Chiapas, los de Gonolobus se conservan en almíbar y sus semillas, hervidas o asadas se comen como las palomitas de maíz. En la región de Otatiltlán, Veracruz, el látex de Gonolobus niger (Cav.) R. Br. ex Schult. se utiliza para producir espuma en una bebida de chocolate. Finalmente, en algunas localidades de los estados de Chiapas y Morelos, el látex de algunas especies de Aspidosperma o Stemmadenia sirve como pegamento escolar.

\section{Materiales y métodos}

La información que aquí se sintetiza proviene de una intensa búsqueda bibliográfica que incluyó trabajos sistemáticos (Woodson, 1935, 1937, 1938a, b, 1941, 1954; Rao, 1956; Gensel, 1969; Stevens, 1988; Leeuwenberg, 1994; Morales, 1995, 1998a, b, 2002a, b, 2005; Henrickson, 1996; Williams, 1996a, b, 1998, 2002; Liede, 1997; Liede y Meve, 1997; Liede y Täuber, 2000; Endress y Bruyns, 2000; Rapini et al., 2003; Morales y Williams, 2004, 2005; Fishbein y Stevens, 2005; Liede y Meve, 2005; Liede-Schumann et al., 2005; Alvarado-Cárdenas y Ochoterena, 2007, Simões et al., 2007) y descripciones de nuevos taxones (Lozada, 2000; Juárez-Jaimes, 2003, 2005; Stevens y Montiel, 2004; Stevens 2005a, b, c; Diego y Lozada, 2006). Entre los tratamientos y listas florísticas consultados están los de Baja California (Wiggins, 1980), Chiapas (Breedlove, 1986), Chihuahua y Sonora (Shreve y Wiggins, 1964; Martin et al., 1998; Felger 2000), Guerrero (Diego, 2004), Michoacán (Rodríguez y Espinosa, 1995), Nuevo León y Tamaulipas (Blackwell, 1964), Nuevo México (Martin y Hutchins, 1981), Oaxaca y Puebla (Juárez-Jaimes y Lozada, 2003; Salas-Morales et al., 2003; Alvarado-Cárdenas, 2004a, b), Querétaro (Argüelles et al., 1991), Quintana Roo (Castillo, 1984) Valle de México (Rzedowski y Rzedowski, 2001), Guatemala (Standley y Williams 1968), Nicaragua (Gentry, 1998) y el sureste de los Estados Unidos de América (Rosatti, 1989). Esta información se complementó con la revisión de ejemplares depositados en los siguientes herbarios nacionales y extranjeros: ENCB, F, FCME, G, MEXU, MO, NY, OAX y XAL. Finalmente se realizó una exploración en algunas bases de datos virtuales (www.mobot.org; www.ny.org; www.mnh.si.edu; www.huh.harvard.edu).

La información analizada sólo se discute en el nivel de especies, aunque las categorías infraespecíficas están indicadas en la lista que se presenta en el Apéndice 1. Para los tipos de vegetación se sigue la propuesta de Rzedowski (1978). Los autores de los nombres científicos se tomaron de Brummitt y Powell (1992).

\section{Resultados}

En la nueva circunscripción de la familia, el número de taxones reconocido para México es de 50 géneros y 385 especies de plantas nativas. Por su número de especies, las Apocynaceae s.l. se ubican en el decimotercer lugar entre las familias con mayor número de especies en México (Cuadro 1).

De las 5 subfamilias reconocidas, 3 están presentes de forma natural en el territorio mexicano, destacando en primer lugar Asclepiadoideae con 22 géneros y 288 especies, seguida por Apocynoideae con 15 géneros y 56 especies y en tercer lugar Rauvolfioideae con 13 géneros y 41 especies (Apéndice 1).

Cuadro 1. Familias con mayor número de especies en México (modificado de Villaseñor, 2003)

\begin{tabular}{ccc}
\hline Familias & Géneros & Especies \\
\hline Asteraceae & 346 & 3021 \\
Poaceae & 166 & 1187 \\
Fabaceae s.l. ${ }^{1}$ & 135 & 1724 \\
Orchidaceae & 157 & 1145 \\
Euphorbiaceae $^{2}$ & 44 & 778 \\
Rubiaceae $^{2}$ & 93 & 593 \\
Cactaceae $^{3}$ & 50 & 550 \\
Lamiaceae $^{\text {Cyperaceae }}$ & 31 & 530 \\
Scrophulariaceae $^{4}$ & 22 & 426 \\
Solanaceae $^{5}$ & 55 & 437 \\
Acanthaceae $^{5}$ & 33 & 430 \\
Malvaceae $^{6}$ & 39 & 400 \\
Apocynaceae s.l. & 52 & 372 \\
Bromeliaceae $^{\text {Verbenaceae }}$ & 50 & 385 \\
Crassulaceae $^{7}$ & 20 & 333 \\
\hline
\end{tabular}

${ }^{1}$ Sousa y Delgado, 1998; ${ }^{2}$ Steinmman, 2002; ${ }^{3}$ Hernández et al. 2004; ${ }^{4}$ Méndez-Larios y Villaseñor-Ríos, 2001; ${ }^{5}$ Daniel, 1999; ${ }^{6}$ Fryxell, 1993; ${ }^{7}$ Willmann et al. 2000. 
Cuadro 2. Géneros, especies y especies endémicas de México en la familia Apocynaceae s.l.

\begin{tabular}{|c|c|c|}
\hline Género & $\begin{array}{l}\text { Total de } \\
\text { especies }\end{array}$ & $\begin{array}{c}\text { Especies } \\
\text { endémicas (\%) }\end{array}$ \\
\hline Allamanda & 1 & 0 \\
\hline Allotoonia & 3 & 0 \\
\hline Amsonia & 4 & 0 \\
\hline Apocynum & 3 & 0 \\
\hline Asclepias & 68 & $33(48)$ \\
\hline Aspidosperma & 2 & 0 \\
\hline Blepharodon & 1 & 0 \\
\hline Cameraria & 2 & 0 \\
\hline Cascabela & 5 & $2(40)$ \\
\hline Cynanchum & 7 & $3(42)$ \\
\hline Dictyanthus & 15 & $13(87)$ \\
\hline Echites & 3 & 0 \\
\hline Fernaldia & 2 & $1(50)$ \\
\hline Fischeria & 1 & 0 \\
\hline Forsteronia & 4 & 0 \\
\hline Funastrum & 11 & $3(27)$ \\
\hline Gonolobus & 39 & $19(49)$ \\
\hline Haplophyton & 2 & 0 \\
\hline Jobinia & 1 & 0 \\
\hline Laubertia & 1 & $1(100)$ \\
\hline Macroscepis & 1 & 0 \\
\hline Mandevilla & 21 & $10(47)$ \\
\hline Marsdenia & 28 & $14(53)$ \\
\hline Matelea & 73 & $44(60)$ \\
\hline Mesechites & 1 & 0 \\
\hline Metalepis & 1 & 0 \\
\hline Metastelma & 25 & $13(52)$ \\
\hline Microdactylon & 1 & $1(100)$ \\
\hline Odontadenia & 1 & 0 \\
\hline Orthosia & 4 & $1(25)$ \\
\hline Oxypetalum & 1 & 0 \\
\hline Pentalinon & 2 & 0 \\
\hline Pherotrichis & 4 & $2(50)$ \\
\hline Plumeria & 2 & 0 \\
\hline Polystemma & 2 & 0 \\
\hline Prestonia & 6 & $1(16)$ \\
\hline Prosthecidiscus & 1 & 0 \\
\hline Rauvolfia & 2 & 0 \\
\hline Rhabdadenia & 1 & 0 \\
\hline
\end{tabular}

Cuadro 2. Continúa

\begin{tabular}{ccc}
\hline Género & $\begin{array}{c}\text { Total de } \\
\text { especies }\end{array}$ & $\begin{array}{c}\text { Especies } \\
\text { endémicas (\%) }\end{array}$ \\
\hline Seutera & 2 & $1(50)$ \\
Stemmadenia & 8 & $3(37)$ \\
Tabernaemontana & 3 & 0 \\
Tassadia & 1 & 0 \\
Thenardia & 3 & $3(100)$ \\
Thevetia & 1 & 0 \\
Thoreauea & 3 & $3(100)$ \\
Tintinnabularia & 2 & $1(50)$ \\
Tonduzia & 2 & 0 \\
Trichosacme & 1 & 0 \\
Vallesia & 7 & $4(57)$ \\
\hline
\end{tabular}

La subfamilia Asclepiadoideae incluye el mayor porcentaje de diversidad, tanto de géneros (44\%) como de especies $(75 \%)$. Cinco géneros contienen poco más de la mitad de las especies, todos ellos de Asclepiadoideae. Asclepias y Matelea son los más ricos, con más de 60 especies (Cuadro 2).

Apocynaceae s.l. presenta su mayor diversidad en las porciones tropicales de los estados ubicados en las vertientes de los océanos Atlántico y Pacífico. Los estados con el mayor número de géneros registrados son Campeche, Chiapas, Guerrero, Oaxaca y Veracruz. Oaxaca es el estado con el mayor número de especies (164), seguido de Chiapas, Veracruz y Guerrero (Cuadro 3) y los que tienen el menor número son Baja California y Tlaxcala.

La familia incluye especies de amplia distribución en México, algunas prácticamente en todas las entidades federativas; entre ellas están Plumeria rubra L. (registrada en 30 estados), Asclepias curassavica L. (27), A. oenotheroides Schltdl. et Cham. (23), A. linaria Cav. (22), Cascabela thevetia (L.) Lippold (22), Vallesia glabra (Cav.) Link (22), Funastrum pannosum (Decne.) Schltr. (20) y F. elegans (Decne.) Schltr. (20).

Las especies de Apocynaceae s.l. se han registrado en casi todos los tipos de vegetación reconocidos en el territorio mexicano. Se encuentran principalmente en el bosque tropical caducifolio (145 especies) y en matorrales xerófilos (122 especies); el bosque de coníferas y el bosque de Quercus en conjunto (bosques templados) integran un porcentaje importante de las especies (106 especies). Los bosques tropicales húmedos ocupan un cuarto lugar, y los bosques espinosos, los pastizales y la vegetación acuática 
Cuadro 3. Géneros y especies de para cada subfamilia de Apocynaceae s.l. en las entidades federativas de México

\begin{tabular}{|c|c|c|c|c|}
\hline \multirow{2}{*}{ Estados } & \multicolumn{4}{|c|}{ Géneros/Especies } \\
\hline & Rauvolfioideae & Apocynoideae & Asclepiadoideae & Totales \\
\hline Aguascalientes & $1 / 1$ & $1 / 1$ & $6 / 15$ & $8 / 17$ \\
\hline Baja California & $2 / 2$ & $1 / 1$ & $5 / 13$ & $8 / 16$ \\
\hline Baja California Sur & $4 / 5$ & $1 / 1$ & $7 / 17$ & $12 / 23$ \\
\hline Campeche & $10 / 15$ & $9 / 12$ & $12 / 28$ & $31 / 55$ \\
\hline Chiapas & $11 / 22$ & $11 / 28$ & $18 / 106$ & $40 / 156$ \\
\hline Chihuahua & $5 / 8$ & $3 / 6$ & $8 / 53$ & $16 / 67$ \\
\hline Coahuila & $3 / 3$ & $2 / 7$ & $7 / 33$ & $12 / 43$ \\
\hline Colima & $7 / 11$ & $6 / 8$ & $10 / 14$ & $23 / 33$ \\
\hline Distrito Federal & $5 / 5$ & $1 / 1$ & $8 / 20$ & $14 / 26$ \\
\hline Durango & $5 / 7$ & $1 / 4$ & $8 / 38$ & $14 / 49$ \\
\hline Guanajuato & $5 / 5$ & $2 / 5$ & $8 / 32$ & $15 / 42$ \\
\hline Guerrero & $11 / 21$ & $9 / 21$ & $13 / 61$ & $33 / 103$ \\
\hline Hidalgo & $4 / 7$ & $2 / 8$ & $9 / 35$ & $15 / 50$ \\
\hline Jalisco & $8 / 16$ & $8 / 13$ & $12 / 66$ & $28 / 95$ \\
\hline Estado de México & $7 / 10$ & $4 / 7$ & $10 / 41$ & $21 / 58$ \\
\hline Michoacán & $8 / 15$ & $7 / 14$ & $13 / 54$ & $28 / 83$ \\
\hline Morelos & $9 / 15$ & $4 / 7$ & $11 / 46$ & $24 / 68$ \\
\hline Nayarit & $9 / 14$ & $4 / 7$ & $11 / 36$ & $24 / 57$ \\
\hline Nuevo León & $1 / 1$ & $2 / 4$ & $9 / 40$ & $12 / 45$ \\
\hline Oaxaca & $11 / 25$ & $11 / 29$ & $19 / 110$ & $41 / 164$ \\
\hline Puebla & $10 / 19$ & $5 / 10$ & $11 / 46$ & $26 / 75$ \\
\hline Querétaro & $6 / 8$ & $6 / 10$ & $9 / 33$ & $21 / 51$ \\
\hline Quintana Roo & $9 / 15$ & $7 / 9$ & $11 / 20$ & $27 / 44$ \\
\hline San Luis Potosí & $6 / 6$ & $5 / 10$ & $10 / 44$ & $21 / 60$ \\
\hline Sinaloa & $9 / 13$ & $3 / 6$ & $10 / 39$ & $22 / 58$ \\
\hline Sonora & $7 / 11$ & $2 / 9$ & $11 / 65$ & $20 / 85$ \\
\hline Tabasco & $10 / 15$ & $6 / 7$ & $8 / 17$ & $24 / 39$ \\
\hline Tamaulipas & $7 / 9$ & $4 / 8$ & $10 / 41$ & $21 / 58$ \\
\hline Tlaxcala & $1 / 1$ & $0 / 0$ & $6 / 11$ & $7 / 12$ \\
\hline Veracruz & $11 / 24$ & $11 / 20$ & $19 / 78$ & $41 / 122$ \\
\hline Yucatán & $10 / 15$ & $7 / 8$ & $11 / 30$ & $28 / 53$ \\
\hline Zacatecas & $4 / 4$ & $1 / 3$ & $8 / 23$ & $13 / 30$ \\
\hline
\end{tabular}

son los tipos de vegetación que registran menos especies (en promedio menos de 40).

El porcentaje de especies endémicas de México alcanza $45 \%$ (176 especies); algunas representan taxones muy atractivos de la flora, como Cascabela thevetioides (Kunth) Lippold y Asclepias schaffnerii A. Gray (Fig. 1). Para México se reconocen 3 géneros endémicos: Microdactylon Brandegee, taxón monoespecífico restringido a la porción 

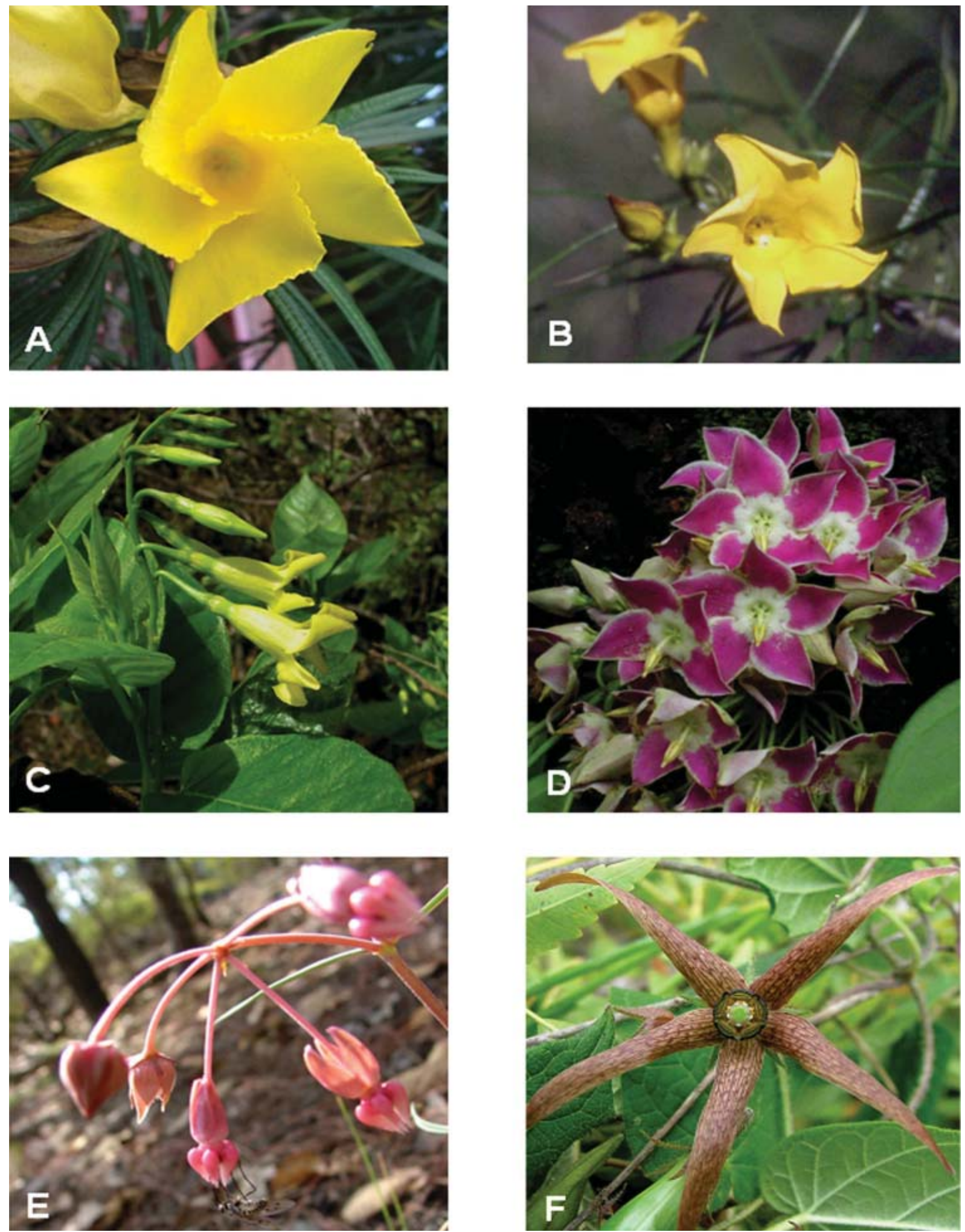

Figura 1. Especies de Apocynaceae s.l. endémicas de México. A.- Cascabela thevetioides, B.- C.pinifolia, C.- Mandevilla convolvulacea, D.- Thenardia floribunda, E.- Asclepias schaffneri, F.- Matelea purpusii. Fotos de L. O. Alvarado-Cárdenas. 
xerofítica del estado de Puebla (Juárez-Jaimes y Lozada, 2003), Thenardia Baill., con 3 especies distribuidas de Jalisco a Chiapas (Williams, 1998) y Thoreauea J. K. Williams, con 3 especies conocidas de Guerrero, Oaxaca y Veracruz (Diego y Lozada, 2006; Morales, 2005; Williams, 2002). La subfamilia Asclepiadoideae aporta el mayor número de especies endémicas ( $83 \%$ del total), donde los géneros con mayor número de taxones endémicos son Asclepias y Matelea, con más de 30 especies y Gonolobus y Marsdenia con 14 o más. De las otras subfamilias, solamente el género Mandevilla (Apocynoideae) tiene 10 especies endémicas; los restantes con endemismos registran de 1 a 4 especies (Cuadro 2).

El estado con mayor número de especies endémicas de México es Oaxaca (68), seguido de Jalisco (51), Guerrero (42) y Michoacán (38). Es importante mencionar que exceptuando Tabasco, en todos los estados de la República Mexicana se registra por lo menos una especie endémica (Fig. 2).

Muchos de los taxones endémicos de México son de amplia distribución (e.g. Dictyanthus pavonii Decne. que se encuentra en 13 estados y Funastrum elegans (Decne.) Schltr. en 20). Sin embargo, algunas especies solamente se conocen de la localidad tipo, y no han sido nuevamente recolectadas y hasta la fecha se consideran de distribución restringida o muy raras; entre ellas se encuentran Asclepias conzattii Woodson, del cañón de Tomellín, Oaxaca; Marsdenia parvifolia Brandegee, de la región de Zapotitlán de las Salinas, Puebla; Thoreauea aberrans J. F. Morales, de Veracruz, y Thoreauea guerrerensis Diego y LozadaPérez, de Guerrero.

Entre los taxones introducidos a la flora de México se encuentran algunas especies de la subfamilia Periplocoideae, así como 12 géneros y 17 especies de otras subfamilias (Apéndice 1). Calotropis procera (Aiton) R. Br., Catharanthus roseus (L.) G. Don, Cryptostegia grandiflora Roxb. ex R. Br., Nerium oleander L. y Vinca major L. son ampliamente cultivadas o ya se encuentran naturalizadas en el país. Hay muchas otras que se han introducido al territorio nacional, cuya distribución se restringe sólo al cultivo, a veces muy local, y para las cuales en ocasiones no existe respaldo en los herbarios, pero han sido observadas por los autores (e.g. Adenium, Hoya, Pachypodium y Stapelia).

La afinidad tropical de la familia se observa al analizar la distribución geográfica de sus géneros presentes en México. Con Centroamérica se comparte más del $80 \%$ de la diversidad genérica y con Sudamérica y las Antillas alrededor del 45\%. Con Norteamérica solamente se comparte el 33\%. Pocos géneros en la flora de México alcanzan una distribución más allá de América; 15\% de ellos se registran en porciones del Viejo Mundo.

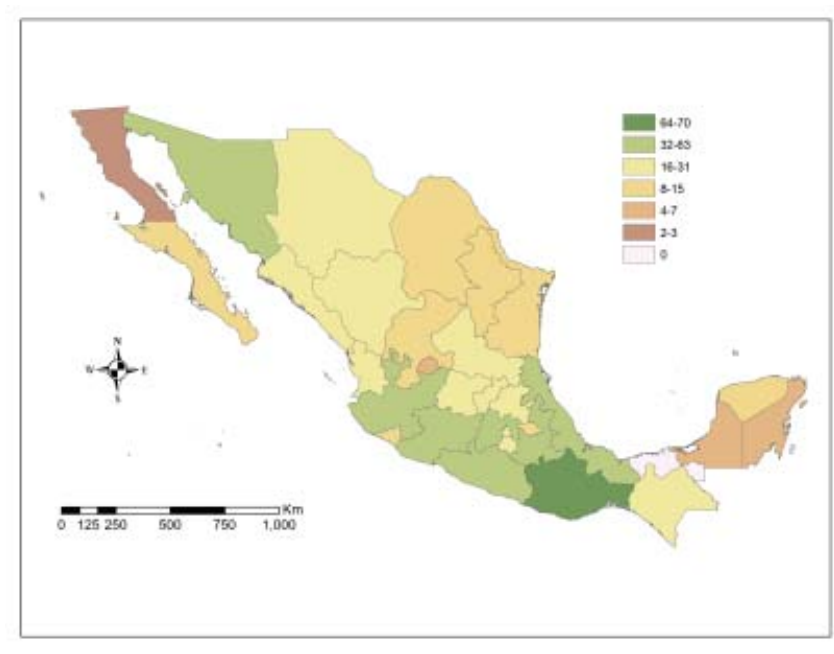

Figura 2. Número de especies endémicas de Apocynaceae s.l. por entidad federativa en México.

En cuanto especies, las Apocynaceae s.l. muestran un patrón ligeramente distinto. Sólo se conocen de México y Centroamérica un total de 146 especies (38\%) y 74 (19\%) se distribuyen de México a Norteamérica. Es interesante observar que sólo 26 especies llegan a Sudamérica y 20 se registran en las islas del Caribe.

\section{Discusión}

Debido a la importancia que tiene el obtener un mejor conocimiento de los recursos biológicos de México, este trabajo representa un primer acercamiento a la diversidad de la familia Apocynaceae en su actual circunscripción taxonómica. Las 385 especies (390 taxones incluyendo los elementos infraespecíficos) sitúan a la familia Apocynaceae como uno de los elementos representativos del paisaje mexicano, ya que es una de las más diversas en el país, ocupando el lugar decimotercero en el nivel específico y el décimo en el genérico. Dentro del orden Gentianales en México, es la familia con más especies y géneros después de Rubiaceae.

Dentro de Apocynaceae, la subfamilia Asclepiadoideae es la mejor representada en México con $75 \%$ de la diversidad específica, aspecto que también se observa en la presencia de especies por estado (Cuadro 3), donde siempre aporta la mayoría de los taxones. Asclepias, Gonolobus y Matelea son ejemplos de géneros que han encontrado en los diferentes hábitats del país los medios para su diversificación, lo que asimismo refleja su amplia tolerancia y adaptación a las distintas condiciones ambientales; por ejemplo, la afilia de especies de Asclepias en zonas áridas. Por otra parte, las 
subfamilias Apocynoideae y Rauvolfioideae no tienen una representatividad tan patente como Asclepiadoideae, pero si tienen un significativo número de especies en el país dentro de los géneros Cascabela, Mandevilla, Thenardia y Vallesia (Cuadro 2). Los resultados sugieren que México es un centro de diversidad para la familia, en particular para Asclepiadoideae; no obstante, estudios comparativos más detallados con otras floras permitirán tener una mejor idea de los centros de diversidad del grupo.

La mayoría de las entidades federativas registran una importante concentración de representantes de Apocynaceae. Sin embargo, la distribución de las especies es particularmente notoria en los estados que colindan con los océanos que bañan su territorio, especialmente Oaxaca, Chiapas, Veracruz y Guerrero, que cuentan con más de un centenar de especies (Cuadro 3).

Apocynaceae se encuentra mejor representada en el bosque tropical caducifolio y el matorral xerófilo. Se han registrado distribuciones similares para otras familias, como Acanthaceae (Daniel, 1998), Burseraceae (Rzedowski et al., 2005) y Euphorbiaceae (Steinnman, 2002). En estos ambientes las Apocynaceae son un componente importante de la estructura de la vegetación; por ejemplo, en el estrato arbóreo Plumeria rubra y Cascabela ovata son elementos típicos de los bosques secos, al igual que los bejucos de varias especies de Gonolobus, Marsdenia y Matelea.

La gran diversidad que presenta la familia, distribuida en casi todo el territorio nacional, concuerda también con su alto porcentaje de endemismo (cercano al 50), similar a otras familias como Asteraceae (Villaseñor, 2003), Lauraceae (Lorea-Hernández, 2002) y Fabaceae (Sousa y Delgado, 1998). La elevada proporción de endemismos es un dato que refuerza la idea de que México es un laboratorio de diversificación de especies. También es cierto que las especies endémicas han sido poco estudiadas, sobre todo aquellas de distribución restringida que pueden estar en peligro de extinción, y de las que poco se sabe sobre sus características genéticas, demográficas y ecológicas, por lo que son deseables análisis más precisos.

Lamayoría de los géneros se distribuyen en elneotrópico, principalmente en Centroamérica, con un número menor hacia las regiones de Norteamérica y el Viejo Mundo. Por otra parte, las especies mexicanas de esta familia restringidas a América presentan 3 grandes componentes: el mesoamericano, donde se encuentra el mayor número de especies y que representa un centro de diversidad para géneros como Cascabela (Alvarado-Cárdenas, 2004b), Mandevilla (Morales, 1998a), Matelea y Stemmadenia; el norteamericano, donde el género Asclepias presenta el mayor número de taxones, y el sudamericano y de la región antillana que comparten con México alrededor del $7 \%$ del total de especies. Previamente Alvarado-Cárdenas
(2003) discutió que Apocynoideae y Rauvolfioideae (Apocynaceae s.str.) comparten más especies con la región mesoamericana que con la sudamericana y el Caribe.

Aunque todavía hay mucho trabajo por desarrollar, las perspectivas a futuro son positivas, ya que el interés por las Apocynaceae ha resurgido y actualmente se encuentran especialistas y estudiantes en todo el mundo investigando el grupo. Es de esperar que la síntesis aquí presentada inspire a otros colegas a estudiar esta familia de morfología tan interesante y compleja.

\section{Agradecimientos}

A los curadores de los herbarios citados en Materiales y métodos, por las facilidades brindadas para la consulta del material. A Mark Fishbein, Mary Endress, Warren D. Stevens, Lucio Lozada, y Rosalinda Medina, sus críticas y sugerencias para mejorar este trabajo. A Enrique Ortiz, la estructura de la base de datos y la elaboración del mapa.

\section{Literatura citada}

Alvarado-Cárdenas, L. O. 2003. La familia Apocynaceae en el Valle de Tehuacán-Cuicatlán. (Puebla-Oaxaca). Tesis Facultad de Ciencias, Universidad Nacional Autónoma de México, México, D.F. 122 p.

Alvarado-Cárdenas, L. O. 2004a. Apocynaceae. Flora del Valle de Tehuacán Cuicatlán 38. Instituto de Biología, Universidad Nacional Autónoma de México, México, D.F. 57 p.

Alvarado-Cárdenas, L. O. 2004b. Las apocináceas. In Biodiversidad de Oaxaca, A. J. García-Mendoza, M. J. Ordoñez y M. Briones-Salas (eds.). Instituto de Biología, Universidad Nacional Autónoma de México, Fondo Oaxaqueño para la Conservación de la Naturaleza y World Wildlife Foundation, México, D.F. p. 171-176.

Alvarado-Cárdenas, L. O. y H. Ochoterena. 2007. A phylogenetic analysis of the Cascabela-Thevetia species complex (Plumerieae, Apocynaceae) based on morphology. Annals of the Missouri Botanical Garden 94:298-323.

Argüelles, E., R. Fernández y S. Zamudio. 1991. Listado florístico preliminar del estado de Querétaro. Flora del Bajío y de Regiones Adyacentes, fascículo complementario II. Instituto de Ecología, Pátzcuaro, Michocán. p. 33-34.

Blackwell Jr., W. H. 1964. Synopsis of the 23 species of Asclepias (Asclepiadaceae) in Tamaulipas and Nuevo León including two new species, Asclepias bifida and 
Asclepias prostrata. The Southwestern Naturalist 9: 171-180.

Breedlove, D. E. 1986. Listados Florísticos de México. IV. Flora de Chiapas. Instituto de Biología, Universidad Nacional Autónoma de México, México, D.F. 246 p.

Brown, R. 1810. On the Asclepiadeae, a natural order of plants separated from the Apocineae of Jussieu. Memoirs of the Wernerian Natural History Society 1: 12-78.

Brummitt, R. K. y C. E. Powell. 1992. Authors of plant names: a list of authors of scientific names of plants, with recommended standard forms of their names, including abbreviations. Royal Botanic Gardens, Kew. $732 \mathrm{p}$.

Castillo A., O. 1984. La familia Apocynaceae en el estado de Quintana Roo, México. Tesis Facultad de Ciencias, Universidad Nacional Autónoma de México, México, D.F. 124 p.

Daniel, T. F. 1999. Acanthaceae. Flora del Valle de Tehuacán-Cuicatlán 23. Instituto de Biología, Universidad Nacional Autónoma de México, 23: 1102.

Daniel, T. F. 1998. Acanthaceae de México: diversidad y distribución. In Diversidad biológica de México: orígenes y distribución, T. P. Ramamoorthy, R. Bye, A. Lot y J. Fa (comps.). Instituto de Biología, Universidad Nacional Autónoma de México, México, D.F. p. 527-544.

Diego, N. 2004. Apocynaceae. Flora de Guerrero 20: 1116.

Diego, N. y L. Lozada. 2006. Thoreauea guerrerensis (Apocynaceae, Apocynoideae), una nueva especie de Guerrero, México. Novon 16: 332-335.

Endress, M. E. 2004. Apocynaceae: Brown and now. Telopea 10: 525-541.

Endress, M. E. y V.P. Bruyns. 2000. A revised classification of the Apocynaceae s.l. Botanical Review (Lancaster) 66: 1-56.

Endress, M. E., B. Sennblad, S. Nilsson, L. Civeyrel, M. W. Chase, S. Huysmans, E. Grafstrom y B. Bremer. 1996. A phylogenetic analysis of Apocynaceae s. str. and some related taxa in Gentianales: a multidisciplinary approach. Opera Botanica Belgica 7: 59-102.

Felger, R. S. 2000. Flora of the Gran Desierto and Río Colorado, Northwestern Mexico. The University of Arizona Press, Tucson. 673 p.

Fishbein, M. and W. D. Stvens. 2005. Resurrection of Seutera Reichenbach (Apocynaceae, Asclepiadoideae). Novon 15: 531-533.

Fryxell, P. A. 1993. Malvaceae. Flora del Valle de Tehuacán-Cuicatlán 1. Instituto de Biología, Universidad Nacional Autónoma de México, México,
D.F. 87 p.

Gensel, W. H. 1969. A revision of the genus Thevetia (Apocynaceae). Master science thesis University of Connecticut, Storrs. 143 p.

Gentry, A. H. 1998. Apocynaceae. In Flora de Nicaragua 85, tomo 1, W. D. Stevens, C. Ulloa U., A. Pool, O.M. Montiel (eds.). Missouri Botanical Garden Press, St. Louis. p. 116-132

Henrickson, J. 1996. Studies in Macrosiphonia (Apocynaceae): generic recognition of Telosiphonia. Aliso 14 (3): 179-195.

Hernández, M. H., C. Gómez-Hinostrosa y B. Goettsch C. 2004. Cactáceas. In Biodiversidad de Oaxaca, A. J. García-Mendoza, M. J. Ordóñez y M. Briones-Salas (eds.). Instituto de Biología, Universidad Nacional Autónoma de México, Fondo Oaxaqueño para la Conservación de la Naturaleza y World Wildlife Foundation, México, D.F. p. 199-207.

Juárez-Jaimes, V. 2003. Especie nueva de Marsdenia (Asclepiadaceae), de Los Tuxtlas, Veracruz, México. Anales del Instituto de Biología, Universidad Nacional Autónoma de México, Serie Botánica 74 (1): 73-78.

Juárez-Jaimes, V. 2005. Una nueva especie de México y Centroamérica, Marsdenia hiriartiana (ApocynaceaeAsclepiadoideae, Marsdenieae). Novon 15: 552-554.

Juárez-Jaimes, V. y L. Lozada. 2003. Asclepiadaceae, Flora del Valle de Tehuacán-Cuicatlán 37. Instituto de Biología. Universidad Nacional Autónoma de México. $57 \mathrm{p}$.

Jussieu, A.L. 1798. Genera plantarum. Herissant, Paris.

Leeuwenberg, A. J. M. 1994. A revision of Tabernaemontana 2. The New World species and Stemmadenia. Wageningen Agricultural University, Royal Botanic Gardens, Kew. 450 p.

Liede, S. 1997. American Cynanchum (Asclepiadaceae)-a preliminary infrageneric classification. Novon 7: 172181.

Liede, S. y U. Meve. 1997. Some clarifications, new species, and new combinations in America Cynanchinae (Asclepiadaceae). Novon 7: 38-45.

Liede, S. y U. Meve 2005. Revision of Metastelma (Apocynaceae-Asclepiadoideae) in Southwestern North America and Central America. Annals of the Missouri Botanical Garden 91: 31-86.

Liede, S. y A. Täuber. 2000. Sarcostemma R. Br. (Apocynaceae-Asclepiadoideae) a controversial generic circumscription reconsidered: evidence from trnl-F spacers. Plant Systematic and Evolution 225: 133-140.

Liede-Schumann, S., A. Rapini, D. J. Goyder y M. W. Chase. 2005. Phylogenetics of the New World subtribes of Asclepiadeae (Apocynaceae-Asclepiadoideae): 
Metastelmatinae, Oxypetalinae and Gonolobinae. Systematic Botany 30: 184-195.

Lorea-Hernández, F. 2002. La familia Lauraceae en el sur de México: Diversidad, distribución y estado de conservación. Boletín de la Sociedad Botánica de México 71: 59-70.

Lozada, L. 2000. Marsdenia gallardoae (Asclepiadaceae), una nueva especie de Chiapas y Oaxaca, México. Novon 10: 128-131.

Martín, P. S., D. Yetman, M. Fishbein, P. Jenkins , T. R. Van Devender y R. K. Wilson. 1998. Gentry's Río Mayo Plants. The tropical deciduous forest and environs of northwest México: Asclepiadaceae. The Southwest Center Series, The University of Arizona Press, Tucson. p. 212-219.

Martin, W. C. y C. R. Hutchins.1981. A Flora of New Mexico. Vol. 2. J. Cramer, Vaduz. p. 1525-1546.

Méndez-Larios, I. y J. L. Villaseñor. 2001. La familia Scrophulariaceae en México: diversidad y distribución. Boletín de la Sociedad Botánica de México 69: 89109.

Morales, J. F. 1995. Evalución del género Alstonia (Apocynaceae) en Centroamérica. Phytologia 78(3): 192-194.

Morales, J. F. 1998a. A synopsis of the genus Mandevilla (Apocynaceae) in Mexico and Central America. Brittonia 50(2): 214-232.

Morales, J. F. 1998b. Three new species and a new combination in Vallesia (Apocynaceae). Novon 8: 263-264.

Morales, J. F. 2002a. Studies in neotropical Apocynaceae: a revision of the genus Laubertia. Rhodora 104: 170185.

Morales, J. F. 2002b. Studies in neotropical Apocynaceae: 2. A review of the genus Fernaldia. Rhodora 104: 186200.

Morales, J. F. 2005. Estudios en las Apocynaceae neotropicales XV: sinopsis del género Thoreauea (Apocynoideae, Echiteae), con una nueva especie de Veracruz, México. Brittonia 57: 258-263.

Morales, J. F. y M. Méndez. 2005. Estudios en las Apocynaceae neotropicales XXII: nuevos realineamientos taxonómicos en el géneroStemmadenia (Apocynaceae, Rauvolfioideae, Tabernaemontanae). Candollea 60: 345-371.

Morales, J. F. y J. K. Williams. 2004. Allotoonia, a new neotropical genus of Apocynaceae based on a subgeneric segregate of Echites. Sida 21: 133-158.

Morales, J. F. y J. K. Williams. 2005. Una nueva combinacion en el género Allotoonia (Apocynaceae, Apocynoideae, Echiteae). Lankesteriana 5: 119-120.

Myers, N., R. A. Mittermeier, G. A. B. da Fonseca y J.
Kent. 2000. Biodiversity hotspots for conservation priorities. Nature 403: 853-858.

Potgieter, Ky V. A. Albert. 2001. Phylogenetic relationships within Apocynaceae s.l. based on trnl intron and trnl-F spacer sequences and propagule characters. Annals of the Missouri Botanical Garden 88: 523-549.

Rao, S. A. 1956. A revision of Rauvolfia with particular reference of the American species. Annals of the Missouri Botanical Garden 43: 253-355.

Rapini, A., M. W Chas, D. J Goyder y J. Griffiths, 2003. Asclepiadeae classification: evaluating the phylogenetic relationships of New World Asclepiadoideae (Apocynaceae). Taxon 52: 33-50.

Rodríguez, L. S. y J. Espinosa. 1995. Listado florístico del estado de Michoacán. Sección I (Gymnospermae; Angiospermae; Acanthaceae- Commelianaceae). Flora del Bajío y de Regiones Adyacentes. Fascículo complementario XV. Instituto de Ecología, A. C., Pátzcuaro, Michoacán. p. 88-100.

Rosatti, J. T. 1989. The genera of the suborder Apocynineae (Apocynaceae and Asclepiadaceae) in the southeastern United States. Journal of the Arnold Arboretum 70: 307-401.

Rzedowski, J. 1978. Vegetación de México. Limusa, México, D.F. 432 p.

Rzedowski, C. G. y J. Rzedowski. 2001. Flora fanerogámica del Valle de México. Instituto de Ecología, Centro Regional del Bajío. Comisión Nacional para el Conocimiento y Uso de la Biodiversidad, Pátzcuaro, Michoacán. 1406 p.

Rzedowski, J., R. Medina y G. Calderón. 2005. Inventario del conocimiento taxonómico, así como de la diversidad y del endemismo regionales de las especies mexicanas de Bursera (Burseraceae). Acta Botanica Mexicana 70: 85-111.

Salas-Morales, S., A. Saynes-Vásquez y L. Schibli. 2003. Flora de la costa de Oaxaca, México: Lista florística de la región de Zimatán. Boletín de la Sociedad Botánica de México 72: 21-58.

Sennblad, B. y B. Bremer. 1996. The familial and subfamilial relationships of Apocynaceae and Asclepiadaceae evaluated with $r b c L$ data. Plant Systematic and Evolution 202: 153-175.

Sennblad, B. y B. Bremer. 2002. Classification of Apocynaceae s.l. according to a new approach combining Linnaean and phylogenetic taxonomy. Systematic Biology 51: 389-409.

Shreve, F. y I. Wiggins 1964. Vegetation and flora of the Sonoran desert. Vol. 2. Stanford University Press, California. p. 1104-1129.

Simões, A. O., L. S. Kinoshita y M. E. Endress. 2007. New combinations and synonyms in Mandevilla Lindley 
(Apocynaceae). Novon 17:87-90.

Simões, A. O., T. Livshultz, E. Conti y M. E. Endress. 2007. Phylogeny and systematics of the Rauvolfioideae (Apocynaceae) based on molecular and morphological evidence. Annals of the Missouri Botanical Garden 94:268-297.

Sousa S., M.y A. DelgadoS. 1998. Leguminosas mexicanas: fitogeografía, endemismo y orígenes. In Diversidad biológica de Mexico: orígenes y distribución, T. P. Ramamoorthy, R. Bye, A. Lot y J. Fa (comps.). Instituto de Biología, Universidad Nacional Autónoma de México, D.F. p. 449-500.

Standley, P. C. 1924. Trees and shrubs of Mexico. Asclepiadaceae. Contributions from the United States National Herbarium 23 (4): 1147-1194.

Standley, P. C. y L. A. Williams 1968. Apocynaceae, Asclepiadeae. In Flora of Guatemala. Fieldiana Botany 24 (8): 334-472.

Steinmann, V. 2002. Diversidad y endemismo de la familia Euphorbiaceae en México. Acta Botanica Mexicana 61: 61-93.

Stevens, W. D. 1988. A synopsis of Matelea subg. Dictyanthus (Apocynaceae: Asclepiadoideae). Annals of the Missouri Botanical Garden 75: 1533-1564.

Stevens, W. D. 2005a. Fourteen new species of Gonolobus (Apocynaceae, Asclepiadoideae) from Mexico and Central America. Novon 15: 222-244.

Stevens, W. D. 2005b. New and interesting milkweeds (Apocynaceae, Asclepiadoideae). Novon 15: 602-619.

Stevens, W. D. 2005c. Novelties in Cynanchum L., sensu Woodson, in Mesoamerica. Novon 15: 620-641.

Stevens, W. D. y O. M. Montiel. 2004. Gonolobus incerianus (Apocynaceae, Asclepiadoideae), Una nueva especie de Mesoamérica. Novon 14: 350-353.

Villaseñor, J. L. 2003. Diversidad y distribución de las Magnoliophyta en México. Interciencia 28: 160-167.

Villaseñor, J. L. 2004. Los géneros de plantas vasculares de la flora de México. Boletín de la Sociedad Botánica de México 75: 105-135.

Wiggins I. L. 1980. Flora of Baja California. Stanford University Press, California. p. 201-209.

Williams, J. K. 1996a. A new combination in Thevetia (Apocynaceae). Sida 17: 185-190.

Williams, J. K. 1996b. The Mexican genera of the Apocynaceae (sensu A. DC.), with key and additional taxonomic notes. Sida 17: 197-213.

Williams, J. K. 1998. A revision of Thenardia H.B.K. (Apocynaceae, Apocynoideae). Lundellia 1: 78-94.

Williams, J. K. 2002. Thoreauea (Apocynaceae: Apocynoideae), a new genus from Oaxaca, Mexico. Lundellia 5: 47-58.

Willmann, D., E. M. Schmidt, M. Heinrich y H. Rimpler. 2000. Verbenaceae. Flora del Valle de TehuacánCuicatlán 27 Instituto de Biología, Universidad Nacional Autónoma de México Mèxico, D.F. 1-75.

Woodson, R. E. 1930. Studies in the Apocynaceae I (a critical studies of the Apocynoidea, with special reference to genus Apocynum). Annals of the Missouri Botanical Garden 17: 1-83.

Woodson, R. E. 1935. Observations on the inflorescence of Apocynaceae. Annals of the Missouri Botanical Garden 22: 2-49.

Woodson, R. E. 1937. New or otherwise noteworthy Apocynaceae of tropical America V. Annals of the Missouri Botanical Garden 24: 14-16.

Woodson, R. E. 1938a. Studies in Apocynaceae. Vll. An evaluation of Plumeria L. and Himatanthus Willd. Annals of the Missouri Botanical Garden 25: 189-224.

Woodson, R. E. 1938b. Apocynaceae. North American Flora. Publications of the New York Botanical Garden 29: 103-192.

Woodson, R.E. 1941. The North American Asclepiadaceae. 1. Perspective of genera. Annals of the Missouri Botanical Garden 28: 193-244.

Woodson, R. E. 1954. The North American species of Asclepias L. Annals of the Missouri Botanical Garden

Apéndice 1. Géneros y especies de Apocynaceae s.l. presentes en México, ordenados por subfamilias.

Entre paréntesis, en abreviatura, las entidades federativas donde se registran.*Especies endémicas de México; + especies introducidas.

\section{Subfamilia Rauvolfioideae}

Allamanda L.

A. cathartica L. (Cam., Chis., Col., D.F., Gro., Méx., Mor., Nay., Oax., Pue., Sin., Tab., Ver., Yuc.)

+ A. schottii Pohl 


\section{Amsonia Walter}

A. grandiflora Alexander (Dgo., Son.)

A. longiflora Torr. (Chih., Coah., Dgo., Son.)

A. palmeri A. Gray (Chih., Son.)

A. tomentosa Torr. et Fremont (Chih.)

Aspidosperma Mart. et Zucc.

A. megalocarpon Müll. Arg. (Cam., Chis., Gro., Oax., Q.Roo, Tab., Ver., Yuc.)

A. spruceanum Benth. ex Müll. Arg. (Cam., Chis., Oax, Q.Roo., Tab.)

\section{Cameraria L.}

C. latifolia L. (Cam, Q.Roo, Tab., Ver., Yuc.)

\section{Carissa L.}

+ C. carandas L.

+C. macrocarpa (Eckl.) A. DC.

\section{Cascabela Raf.}

C. gaumeri (Hemsl.) Lippold (Cam., Gro., Q.Roo, Tab., Tamps., Ver., Yuc.)

C. ovata (Cav.) Lippold (BCS., Chis., Col., Dgo., Gro., Hgo., Ja.l, Mex., Mich., Mor., Nay., Oax., Pue., Sin., Tab., Ver., Zac.)

*C. pinifolia (Standl. et Steyerm.) Alvarado-Cárdenas et Ochot.-Booth (Gro., Mich., Pue.)

C. thevetia (L.) Lippold (Cam., Chis., Chih., Gto., Gro., Hgo., Jal., Méx., Mich., Mor., Nay., Oax., Pue., Qro, Q.Roo., S.L.P., Sin., Son., Tab., Tamps, Ver., Yuc.)

${ }^{*}$ C. thevetioides (Kunth) Lippold (D.F., Gto., Gro., Hgo., Jal., Méx., Mich., Mor., Oax., Pue., Qro., Sin., Tamps.)

\section{Catharanthus G. Don}

+C. roseus (L.) G. Don

Haplophyton A. DC.

H. cimicidum A. DC. (Chis., Col., Gro., Jal., Méx., Mich., Mor., Oax., Pue., Ver.)

H. crooksii (L. Benson) L. Benson (Chih., Coah., Sin., Son.)

\section{Plumeria L.}

P. obtusa L. variedad sericifolia (C. Wright ex Griseb.) Woodson (Cam., Q.Roo., Ver., Yuc.)

P. rubra L. forma acutifolia (Poir.) Woodson (Ags., B.C.S., Cam., Chis., Chih., Dgo., Gro., Hgo, Jal., Méx., Mich., Mor., Nay., N.L., Oax., Pue., Qro., Q.Roo., Sin., Son., Tab., Tamps., Ver., Yuc., Zac.)

P. rubra L. forma rubra (Ags., Cam., Chis., Col., D.F., Gro., Hgo., Jal., Méx., Mich., Mor., Nay., Oax., Pue., Qro., Tab., Tamps., Ver., Yuc.)

\section{Rauvolfia L.}

R. ligustrina Willd. ex Roem. et Schult. (Chis., Gro., Jal., Oax., Pue., Q.Roo, Ver., Yuc.)

R. tetraphylla L. (Cam., Chis., Col., Gto., Gro., Jal., Méx., Mich., Mor., Nay., Oax., Pue., Qro., Q.Roo., S.L.P., Sin., Tab., Tamps., Ver., Yuc.)

\section{Stemmadenia Benth.}

S. donnell-smithii (Rose) Woodson (Cam., Chis., Col., Dgo., Gro., Hgo., Jal., Mich., Mor., Nay., Oax,. Pue., Qro., Q.Roo, S.L.P., Sin., Tab., Ver.) 
S. eubracteata Woodson (Chis., Gro., Oax., Ver.)

S. hannae M. Méndez et J. F. Morales (Chis.)

S. litoralis (Kunth) L. Allorge (Cam., Chis., Col., Hgo., Mor., Nay., Oax., Pue., Tab., Tlax., Ver., Yuc.)

S. pubescens Benth. (Chih., Dgo., Gro., Jal., Méx., Mich., Mor., Nay., Oax., Pue., Ver.)

*S. stenoptera Leuweenb. (Col., Jal.)

*S. tomentosa Greenm. (Chih., Col., Gro., Jal., Méx., Mich., Mor., Nay., Oax., Pue., Sin., Son., Ver., Zac.)

*S. venusta J. F. Morales (Oax.)

\section{Tabernaemontana L.}

T. alba Mill. (Cam., Chis., Mich., Oax., Pue., Qro., Q.Roo., S.L.P., Tab., Tamps., Ver., Yuc.)

T. amygdalifolia Jacq. (Cam., Chis., Dgo., Gro., Jal., Mich., Nay., Oax., Pue., Qro., Q.Roo, Sin., Verr, Yuc., Zac.)

T. arborea Rose (Chis., Tab., Ver.)

+ T. divaricata (L.) R. Br. ex Roem. et Schult.

\section{Thevetia L.}

T. ahouai (L.) A. DC. (Cam., Chis., D.F., Gto., Gro., Mor., Nay., Oax., Pue., Q.Roo., Sin, Son., Tab., Tamps., Ver., Yuc.)

\section{Tonduzia Pittier}

T. longifolia (A. DC.) Markgr. (Chis., Col., Gro., Jal., Mich., Mor., Nay., Oax., Pue., Ver.)

T. stenophylla (Donn. Sm.) Pittier (Chis., Col., Gro., Jal., Mex., Mich., Mor., Nay., Oax., Pue.)

Vallesia Ruiz et Pav.

V. antillana Woodson (Q.Roo, Tamps., Ver., Yuc.)

V. aurantiaca (M. Martens et Galeotti) J.F. Morales (Chis., Gro., Jal., Oax., Ver.)

*V. baileyana Woodson (Son.)

V. glabra (Cav.) Link (B.C., B.C.S., Cam., Chis., D.F., Gto., Gro., Hgo., Mich., Mor., Nay., Oax., Pue., Qro., Q.Roo, S.L.P., Sin., Son., Tab., Tamps., Ver., Yuc.)

*V. laciniata Brandegee (B.C.S., Son.)

V. sinaloensis El. Mey. ex J. F. Morales (Sin.)

*V. spectabilis El. Mey. ex J. F. Morales (Jal.)

\section{Vinca L.}

+ V. major L.

+ V. minor L.

\section{Subfamilia Apocynoideae}

Allotoonia J. F. Morales et J. K. Williams

A. turbinata (Woodson) J. F. Morales et J. K. Williams (Cam., Chis.)

A. tuxtlensis (Standl.) J. F. Morales et J. K. Williams (Cam., Chis., Oax., Qro., Q.Roo, S.L.P., Ver., Yuc.)

A. woodsoniana (Monach.) J. F. Morales et J. K. Williams (Gro., Jal., Mich., Oax.)

Adenium Roem. et Schult.

+ A. obesum (Forssk.) Roem. et Schult.

Apocynum L.

A. androsaemifolium L. (Chih., Coah., Son.) 
A. cannabinum L. (B.C., Chih., Coah., N.L., Qro., Son., Tamps.)

A. sibiricum Jacq. (Coah., Son.)

Beaumontia Wall.

+ B. grandiflora Wall.

Echites P. Browne

E. turriger Woodson (Col., Gro., Jal., Mich.)

E. umbellatus Jacq. (Cam., Q.Roo., Tab., Ver., Yuc.)

E. yucatanensis Millsp. (Cam., Q.Roo, Yuc.)

Fernaldia Woodson

*F. asperoglostis Woodson (Gro., Méx., Mich.)

F. pandurata (A. DC.) Woodson (Cam., Chis., Col., Gro., Jal., Oax., Pue., Qro., Q.Roo, S.L.P., Sin., Tamps., Ver., Yuc.)

Forsteronia G. Mey.

F. acouci (Aubl.) A.DC. (Chis., Oax., Ver.)

F. myriantha Donn. Sm. (Chis., Gro., Oax., Pue., Ver.)

F. peninsularis Woodson (Cam. Chis.)

F. spicata (Jacq.) G. Mey. (Chis., Gro., Jal., Nay., Oax., Ver.)

Laubertia A. DC.

*L. contorta (M. Martens et Galeotti) Woodson (Chih., Col., Gro., Jal., Méx., Mich., Mor., Oax., Pue., Sin.)

Mandevilla Lindl.

M. acutiloba (A. DC.) Woodson (Chis., Gro., Oax.)

*M. andrieuxii (Müll. Arg.) Hemsl. (Col,. Jal., Mich., Oax.)

M. brachysiphon (Torr.) Pichon (Chih., Dgo., Son.)

*M. convolvulacea (A. DC.) Hemsl. (Oax., Pue.)

*M. foliosa (Müll. Arg.) Hemsl. (Chih., Col., D.F., Dgo., Gto., Gro., Hgo., Jal., Méx., Mich., Mor., Nay., Oax,. Pue., Qro., Sin., Son., Ver.)

*M. hesperia (I. M. Johnst.) A. Simões, L. S. Kinoshita-Gouvêa et M. Endress (B.C.S., Son.)

M. hirsuta (A. Rich.) K. Schum. (Chis., Oax.)

*M. holosericea (Sessé et Moc) J. K. Williams (Chis., Col., Gto., Gro., Hgo., Jal., Méx., Mich., Mor., Nay., Oax., Pue., Qro., Sin., Ver.)

M. hypoleuca (Benth.) Pichon (Ags., Coah., Dgo., Gto., Gro., Hgo., Jal., Méx., Mich., Mor., Nay., N.L., Pue., Qro., S.L.P., Sin., Son., Tamps., Zac.)

*M. karwinskii (Müll. Arg.) Hemsl. (Coah., Gto., Hgo., Méx., N.L., Qro., S.L.P., Tamps., Ver., Zac.)

M. lanuginosa (M. Martens et Galeotti) Pichon variedad lanuginosa (Coah., Hgo., N.L., Pue., S.L.P, Tamps.)

*M. lanuginosa (M. Martens et Galeotti) Pichon variedad oaxacensis Henr. (Gro., Oax., Pue.)

M. macrosiphon (Torr.) Pichon (Chih., Coah., Dgo., S.L.P., Son., Tamps., Zac.)

*M. mexicana (Müll. Arg.) Woodson (Oax.)

*M. nacapulensis (Felger et Henr.) A. Simões, L. S. Kinoshita-Gouvêa et M. Endress (Son.)

*M. oaxacana (A. DC.) Hemsl. (Oax.) 
*M. pringlei J. K. Williams (Jal., Mich.)

M. subsagittata (Ruiz et Pav.) Woodson (Cam., Chis., Gro., Hgo., Jal., Mich., Mor., Nay., Oax., Pue., Qro., Q.Roo, S.L.P., Sin., Tab., Tamps., Ver., Yuc.)

M. subsessilis (A. DC.) Woodson (Chis., Gro., Mich., Oax., S.L.P.)

M. torosa (Jacq.) Woodson (Cam., Chis., Hgo., S.L.P.)

M. tubiflora (M. Martens et Galeotti) Woodson (Chis., Gro., Mich., Oax., Ver.)

M. villosa (Miers) Woodson (Chis.)

Mesechites Müll. Arg.

M. trifidus (Jacq.) Müll. Arg. (Cam., Chis., Oax., Tab., Tamps., Ver.)

Nerium L.

$+N$. oleander L.

Odontadenia Benth.

O. macrantha (Roem. et Schult.) Markgraf (Chis., Oax.)

Pachypodium Lindl.

$+P$. lamerei Drake

+ P. saundersii N. E. Br.

Pentalinon Voigt

P. andrieuxii (Müll. Arg.) B. F. Hansen et Wunderlin (Cam., Chis., Hgo., Oax., Pue., Qro., Q.Roo, S.L.P., Tab., Ver., Yuc.)

P. luteum (L.) B. F. Hansen et Wunderlin (Chis.)

Prestonia R. Br.

*P. clandestina J. F. Morales (Chis., Ver.)

P. longifolia (Sessé et Moc.) J. F. Morales (Chis.)

P. mexicana A. DC. (Cam., Chis., Col., Gto., Gro., Jal., Mich., Mor., Nay., Oax., Qro., Q.Roo, S.L.P., Tab., Ver.)

P. portobellensis (Beurl.) Woodson (Chis., Oax., Q.Roo, Tab., Ver., Yuc.)

P. speciosa Donn. Sm. (Chis., Oax., Ver.)

Rhabdadenia Müll. Arg.

R. biflora (Jacq.) Müll. Arg. (Cam., Chis., Q.Roo, Tab., Ver., Yuc.)

Thenardia Kunth

*T. chiapensis J. K. Williams (Chis., Gro., Oax.)

*T. floribunda Kunth (Col., Gro., Jal., Méx., Mich., Mor., Nay., Oax.)

*T. galeottiana Baill. (Chis., Gro., Oax.)

Thoreauea J. K. Williams

*T. aberrans J. F. Morales (Ver.)

*T. guerrerensis Diego et Lozada-Pérez (Gro.)

*T. paneroi J. K. Williams (Gro., Oax.)

Tintinnabularia Woodson

*T. gratissima J. F. Morales (Ver.)

T. mortonii Woodson (Chis.) 
Trachelospermum Lem.

$+T$. difforme (Walter) A. Gray

\section{Subfamilia Periplocoideae}

Cryptostegia R. Br.

+ C. grandiflora Roxb. ex R. Br.

+ C. madagascarensis Bojer ex Decne.

\section{Subfamilia Asclepiadoideae}

\section{Asclepias L.}

A. albicans S. Watson (B.C., B.C.S., Son.)

A. angustifolia Schweigg. (Chis., Chih., D.F., Dgo., Gro., Hgo., Jal., Méx., Mich., Nay., N.L.., Oax., Qro., S.L.P., Son., Tamps., Ver., Zac.)

A. arenaria Torr. (Chih., Dgo.)

A. asperula (Decne.) Woodson (Chih., Coah., Dgo., N.L., S.L.P., Son., Tamps.)

*A. atroviolacea Woodson (Chih., Dgo., Son.)

A. auriculata Kunth (Chis., Gro., Jal., Méx, Mich., Mor., Nay., Oax., Pue., Ver., Zac.)

A. brachystephana Engelm. ex Torr. (Ags., Chih., Coah., Dgo., Gto., S.L.P., Son., Zac.)

*A. circinalis (Decne.) Woodson (Gro., Méx., Oax., Pue.)

A. contrayerba Sessé et Moc. (Ags., Chis., Chih., Dgo., Gto., Gro., Hgo., Jal., Méx., Mich., Mor., Nay., N.L., Oax., Pue., Qro., Sin., Tab., Tamps., Ver.)

*A. conzattii Woodson (Oax.)

*A. coulteri A. Gray (Gto., Hgo., Qro., S.L.P., Tamps.)

*A. crocea Woodson (Jal.)

A. curassavica L. (Ags., B.C., B.C.S., Cam., Chis., Chih., Col., Gto., Gro., Hgo., Jal., Méx., Mich., Mor., Nay., N.L., Oax., Pue., Qro., Q.Roo, Sin., Son., Tab., Tamps., Ver., Yuc., Zac.)

A. elata Benth. (Chih., Coah., N.L., Son., Tamps.)

A. emoryi (Greene) Vail ex Small (N.L., Tamps.)

A. engelmanniana Woodson (Coah., Dgo., Son.)

A. eriocarpa Benth. (B.C.)

A. erosa Torr. (B.C., Son.)

*A. euphorbiifolia Engelm. ex A. Gray (Dgo., S.L.P.)

A. fascicularis Decne. (B.C.)

*A. fournieri Woodson. (Chih., Hgo., Jal., Méx., Mich., Nay., Qro., Son.)

*A. gentryi Standl. (Chih, Son.)

A. glaucescens Kunth (Ags., Chis., Dgo., Gro., Jal., Méx., Mich., Mor., Nay., N.L., Oax., Pue., Sin., Son., Tlax., Ver.)

A. hypoleuca (A. Gray) Woodson (Chih., Son.)

A. involucrata Engelm. ex Torr. (Chih., Coah., Dgo., Son.)

A. jaliscana B. L. Rob. (Chih., Gro., Jal., Oax., Pue., Son.)

*A. jorgeana Fishbein et S. P. Lynch (Chih., Dgo., Gto., Son.)

*A. laxiflora Colla (Oax.) 
A. lemmonii A. Gray (Chih., Dgo., Jal., Son.)

*A. leptopus I. M. Johnst. (Chih., Sin., Son.)

A. linaria Cav. (Ags., Chih., Coah., D.F., Dgo., Gto., Gro., Hgo., Jal., Méx., Mich., Mor, N.L., Oax., Pue., Qro., S.L.P., Son., Tamps., Tlax., Ver., Zac.)

*A. lynchiana Fishbein (Gro., Jal., Méx., Mich., Mor., Oax., Pue.)

A. macrotis Torr. (Chih, Coah.)

*A. macroura A. Gray (Gto., Jal., Nay.)

*A. masonii Woodson (B.C.S.)

*A. mcvaughii Woodson (Gro., Jal.)

*A. mexicana Cav. (Coah., D.F., Gto, Hgo., Méx., Mich., N.L., Oax., Pue., S.L.P., Tamps., Ver.)

*A. mirifica Woodson (Chih., Son.)

*A. notha W. D. Stevens (Ags., Coah., D.F., Dgo., Hgo., Jal., Méx., Mich., Mor., Nay., Oax., Pue., S.L.P., Tlax., Ver.) A. nummularia Torr. (Chih, Coah., Dgo., Gto, S.L.P., Son.)

*A. nummularioides W. D. Stevens (Gto., Méx., S.L.P.)

A. oenotheroides Schltdl. et Cham. (Ags., Cam., Chis., Chih., Coah., Dgo., Gto., Gro.., Hgo., Jal., Méx., Mich., Mor., N.L., Oax., Pue., Qro., S.L.P., Son., Tab., Tamps., Ver., Yuc.)

*A. otarioides E. Fourn. (Coah., D.F., Dgo., Gto., Hgo., Jal., Méx., Mich., N.L., Oax., Qro., S.L.P., Tlax., Ver., Zac.)

*A. ovata M. Martens et Galeotti (Chih., Col., D.F., Dgo., Gro., Hgo., Jal., Méx., Mich., Mor., Nay., Qro., Sin., Son., Tamps., Ver.)

A. pellucida E. Fourn. (Chis., Gto., Gro., Hgo., Jal., Oax., Qro., S.L.P., Ver.)

*A. pratensis Benth. (Gto., Jal., Mich., Nay.)

*A. pringlei (Greenm.) Woodson (D.F., Dgo., Gto., Gro., Hgo., Jal., Méx., Mich., Mor., Nay., Oax., Pue., S.L.P., Ver.)

*A. prostrata W. H. Blackw. (Tamps.)

*A. pseudorubricaulis Woodson (S.L.P.)

*A. puberula A. Gray (Ags., Dgo., Hgo., Jal., Méx., S.L.P., Ver.)

A. quinquedentata A. Gray (Ags., Chih., D.F., Hgo., Méx., Mich., S.L.P., Son.)

A. rosea Kunth (Chis., Dgo., Gto., Gro., Jal., Méx., Mich., Mor., Oax., Ver., Zac.)

A. scaposa Vail (Coah., N.L., Pue., S.L.P., Zac.)

*A. schaffneri A. Gray (Gto., S.L.P., Zac.)

*A. scheryi Woodson (Mich., Nay.)

A. similis Hemsl. (Chis., Gto., Gro., Mich., N.L., Oax., Qro., S.L.P., Tamps., Ver.)

A. sperryi Woodson (Coah.)

*A. standleyi Woodson (Sin., Son.)

*A. subaphylla Woodson (Sin., Son.)

A. subulata Decne. (B.C., B.C.S., Sin., Son.)

A. subverticillata (A. Gray) Vail (Ags., Chih., Coah., Dgo., N.L., Qro., S.L.P., Son.)

A. texana A. Heller (Chih., Coah., N.L.)

A. tuberosa L. (Chih., Coah., N.L., Son., Tamps.)

*A. vinosa (E. Fourn.) Woodson (D.F., Mor., Oax.) 
A. viridiflora Raf. (Coah., N.L.)

*A. virletii E. Fourn. (Coah., N.L., S.L.P.)

A. woodsoniana Standl. et Steyerm. (Chis., Oax., Ver.)

*A. zanthodacryon (L. B. Sm.) Woodson (Coah., N.L.)

Blepharodon Decne.

B. mucronatum (Schltdl.) Decne. (Cam., Chis, Col., Gro., Hgo., Jal., Mich., Mor., Nay., Oax., Qro, Q.Roo, S.L.P., Tab., Ver., Yuc.)

Calotropis R. Br.

+ C. procera (Aiton) R. Br.

Ceropegia L.

+ C. woodi Schltr.

Cynanchum L.

C. cyathiforme (Sundell) W. D. Stevens (Chis.)

${ }^{*}$. foetidum (Cav.) Kunth (Chis., D.F., Gto., Gro., Jal., Méx., Mich., Mor., Oax., Pue., Qro., Tlax., Yuc.)

${ }^{*}$ C. jaliscanum (Vail.) Woodson (Col., Jal., Mich., Nay)

*C. ligulatum (Benth.) Woodson (Ags., Chih., Dgo., Gro., Jal., Méx., Mich., Mor., Nay., Qro., Sin., Son., Zac.)

C. rensonii (Pittier) Woodson (Cam., Yuc.)

C. surrubiflorum W. D. Stevens (Tab., Ver.)

C. unifarium (Scheele) Woodson (Cam., Chih., Coah., Gro., Jal., N.L., Oax., Qro., Sin., Tamps., Ver., Yuc.)

*Dictyanthus Decne.

*D. aeneus Woodson (Yuc.)

*D. altatensis (Brandegee) W. D. Stevens (Sin., Son.)

D. asper (Mill.) W. D. Stevens (Chis, Gro., Jal., Mich., Oax., Pue., Ver.)

*D. eximius (W. D. Stevens) W. D. Stevens (Chis.)

*D. hamatus (W. D. Stevens) W. D. Stevens (Gro., Oax.)

*D. lautus (W. D. Stevens) W. D. Stevens (Col.)

*D. macvaughianus (W. D. Stevens) W. D. Stevens (Jal., Mich.)

D. parviflorus Hemsl. (Ags., Chis, D.F., (Dgo., Jal., Méx., Mich., Mor., Oax., Ver.)

*D. pavonii Decne. (Col., Dgo., Gto., Gro., Jal., Méx., Mich., Mor., Nay, Oax., Sin., Ver., Zac.)

*D. reticulatus (Turcz.) Benth et Hook. f. ex Hemsl. (Gro., Jal., Méx., Mor., Nay., Oax., Pue., Sin.)

*D. sepicola (W. D. Stevens) W. D. Stevens (Chih., Jal., Nay., Son.)

*D. suffruticosus (W. D. Stevens) W. D. Stevens (Oax.)

*D. tigrinus Conzatti et Standl. (Chis., Oax., Ver.)

*D. tuberosus B. L. Rob. (Jal., Nay., Sin., Son.)

*D. yucatanensis Standl. (Cam., Chis., Q.Roo, Ver., Yuc.)

Fischeria DC.

F. scandens DC. (Cam., Chis., Oax., Tab., Ver.)

Funastrum E. Fourn.

*F. arenarium (Decne. ex Benth.) Liede (B.C.) 
F. bilobum (Hook. et Arn.) J. F. Macbr. (Cam., Chis., Gro., Jal., Mich., Mor., Oax., Pue., Ver., Yuc.)

F. clausum (Jacq.) Schltr. (B.C.S., Cam., Chis., Chih, Col., Dgo., Gro., Jal., Mich., Mor., Nay., Oax., Qro., Q.Roo, Sin., Son., Tab., Tamps., Ver., Yuc.)

F. crispum (Benth.) Schltr. (Ags., B.C., Chih., Coah., Dgo., Jal., N.L., Qro, S.L.P., Son., Tamps., Zac.)

F. cynanchoides (Decne.) Schltr. variedad cynanchoides (Chih., Coah., Dgo., N.L., Sin., Son., Tamps.)

F. cynanchoides (Decne.) Schltr. variedad hartwegii (Vail) Krings (Chis.,Chih., Coah., Dgo., Gto., Jal., Nay., Qro, Sin., Son., Zac.)

*F. elegans (Decne.) Schltr. (Ags., Cam., Chis., Coah., D.F., Gto., Gro., Hgo., Jal., Méx., Mich., Mor., Oax., Pue., Qro, S.L.P., Sin., Tlax., Ver., Yuc.)

F. hirtellum (A. Gray) Schltr. (B.C.)

F. lindenianum (Decne.) Schltr. (Chis., Ver., Yuc.)

F. odoratum (Hemsl.) Schltr. (Cam., Chis.)

*F. pannosum (Decne.) Schltr. (Ags., B.C.S., Chih., Col., Dgo., Gto., Gro., Jal., Méx., Mich., Mor., Nay., Oax., Pue., Qro., S.L.P., Sin., Son., Tamps., Zac.)

F. torreyi (A. Gray) Schltr. Chih., Coah., Dgo., Gto., N.L., Tamps., Zac.)

Gomphocarpus R. Br.

+ G. fruticosus (L.) W. T. Aiton

+ G. physocarpus E. Mey.

Gonolobus Michx.

${ }^{*}$ G. albiflorus W. D. Stevens (Jal.)

G. ancorifer W. D. Stevens (Chis., Oax., Ver.)

G. aristolochiifolius (Brandegee) Woodson (Chis.)

G. arizonicus (A. Gray) Woodson (Son.)

G. barbatus Kunth (Cam., Chis., Gro., Mich., Mor., Nay., Oax., Pue., Q.Roo, Sin., Tab., Ver., Yuc.)

*G. bifidus Hemsl. (S.L.P.)

*G. breedlovei L. O. Williams (Chis.)

G. chiapensis (Brandegee) Woodson (Chis.)

${ }^{*}$ G. chloranthus Schltdl. (Chih., Gto., Gro., Jal. Hgo. Oax., Pue., Qro.,Ver.)

*G. croceus W. D. Stevens (Nay., Sin.)

G. cteniophorus (S. F. Blake) Woodson (Cam., Chis., Oax., Tab., Ver., Yuc.)

G. cuajayote W. D. Stevens (Chis.)

G. dasystephanus (S. F. Blake) Woodson (Chis.)

G. erianthus Decne. (Chis., D.F., Gto., Gro., Hgo., Jal., Mich., Mor., Nay., N.L., Oax., Qro, Tamps., Ver.)

G. exannulatus W. D. Stevens (Chis.)

G. fraternus Schltdl. (Cam., Chis., Oax., S.L.P., Tab., Tamps., Ver.)

*G. fuscus Decne. (Mich.)

*G. gonoloboides (Greenm.) Woodson (Chih., Dgo., Sin., Son.)

*G. grandiflorus (Cav.) R. Br. ex Roem. et Schult. (Gto., Gro., Hgo., Jal., Mich., Mor., Oax., Pue., Qro., Sin., Ver., Zac.) 
G. incerianus W. D. Stevens et Montiel (Chis., Oax., Ver.)

*G. jaliscensis B. L. Rob. et Greenm. (Gro., Jal., Mich., Mor., Nay., Oax.)

G. leianthus Donn. Sm. (Cam., Chis., Tab., Ver.)

*G. luridus Decne. (Ver.)

*G. megalocarpus Paul G. Wilson (Méx.)

*G. nemorosus Decne. (Oax., Pue., Ver.)

G. niger (Cav.) R. Br. ex Schult. (Chis., Gro., Hgo., Oax., Qro, S.L.P., Tab., Tamps., Ver.)

*G. pallidus W. D. Stevens (Chis.)

*G. pectinatus Brandegee (Gro., Méx., Mor., Oax., Pue., Ver.)

G. sandersii W. D. Stevens (Col., Jal.)

*G. sidaefolius M. Martens et Galeotti (Ver.)

${ }^{*}$ G. sororius A. Gray (Gro., Jal., Oax.)

G. stenanthus (Standl.) Woodson (Cam., Q.Roo, Yuc.)

G. stenosepalus (Donn. Sm.) Woodson (Chis., Ver.)

*G. striatus M. Martens et Galeotti (Hgo.)

*G. tingens Decne. (Hgo.)

*G. triflorus M. Martens et Galeotti (Oax.)

G. uniflorus Kunth (Ags., Chis., D.F., Gto., Gro., Jal., Méx., Mich., Mor., Oax., Pue., Qro, Tamps., Tlax.)

G. versicolor Woodson (Chis.)

G. xanthotrichus Brandegee (Chis., Oax., Ver.)

Hoya R. Br.

$+H$. carnosa R. Br.

Huernia R. Br.

$+H$. macrocarpa Schweinfurth ex K. Schum.

Jobinia E. Fourn.

J. eulaxiflora (Lundell) W. D. Stevens (Ver.)

Macroscepis Kunth

M. diademata (Ker Gawl.) W. D. Stevens (Cam., Chis., Gro., Mich., Nay., Oax., Q.Roo, S.L.P. Sin., Son., Tamps., Ver., Yuc.)

M. pleistantha Donn. Sm. (Chis.)

Marsdenia R. Br.

*Marsdenia astephanoides (A. Gray) Woodson (Gro., Jal., Mich., Nay., Oax., Sin.)

M. bourgaeana (Baill.) W. Rothe (Chis., Gro., Méx., Mor., Oax., Ver.)

*M. callosa Juárez-Jaimes et W. D. Stevens (Gro., Mich., Oax.)

*M. carterae W. D. Stevens et Juárez-Jaimes (B.C.S.)

*M. coulteri Hemsl. (Cam., Chis., Coah., Gto., Gro., Hgo., Jal., N.L., Oax., Pue., Qro., Q.Roo, Sin., Son., Tamps., Ver., Yuc.)

*M. edulis S. Watson (Nay., Sin., Son.)

*M. gallardoae Lozada-Pérez (Chis., Oax.) 
M. gualanensis Donn. Sm. (Cam., Oax., Yuc.)

M. gymnemoides W. Rothe (Chis.)

M. hiriartiana Juárez-Jaimes et W. D. Stevens (Chis.)

M. lanata (Paul G. Wilson) W. D. Stevens (Chis., Gro., Jal., Mich., Nay., Oax., Pue., Sin.)

M. laxiflora Donn. Sm. (Chis., Tab., Ver., Yuc.)

M. mayana Lundell (Chis.Gro., Qroo., Yuc.)

M. mexicana Decne. (Chis., Gro., Jal., Méx., Mich., Mor., Oax., Pue., Ver.)

M. neriifolia (Decne.) Woodson (Chis., Oax., Ver.)

*M. oaxacana Morillo (Oax.)

*M. parvifolia Brandegee (Oax., Pue.)

*M. popoluca Juárez-Jaimes et A. Campos (Ver.)

*M. pringlei S. Watson (N.L., Qro., S.L.P., Tamps.)

M. propinqua Hemsl. (Cam., Chis., Col., Gro., Hgo., Mich., N.L., Oax., Q.Roo, Tab., Tamps., Ver., Yuc.)

*M. purpusiana W. D. Stevens (Ver.)

*M. rzedowskiana Juárez-Jaimes et W. D. Stevens (Oax., Pue., Ver.)

M. schlechteriana W. Rothe (Chis.)

M. stephanotidifolia Woodson (Chis.)

M. steyermarkii Woodson (Chis.)

M. trivirgulata Bartlett (Chis., Col., Gro., Jal., Mich., Oax.)

*M. tubularis L. O. Williams (Chis., Méx.)

*M. zimapanica Hemsl. (Gro., Hgo., Jal., Méx., Mich., Mor., Nay., Oax., Pue., Qro.)

Matelea Aubl.

*M. adenocardia (Standl.) Woodson (Chih.)

${ }^{*} M$. angustiloba (B. L. Rob. et Greenm.) W.D. Stevens (Nay.)

*M. atrocoronata (Brandegee) Woodson (Oax., Pue.)

M. belizensis (Lundell et Standl.) Woodson (Q.Roo, Yuc.)

*M. calcarata Woodson (N.L., S.L.P., Tamps.)

*M. calcicola (Greenm.) Woodson (Gro., Mor., Oax.)

M. campechiana (Standl.) Woodson (Cam., Chis., Q.Roo, Yuc.)

$*$ M. camporum (Brandegee) Woodson (Coah.)

*M. castanea (Brandegee) Woodson (Oax.)

*M. caudata (A. Gray) Woodson (Chih., Mor., Oax., Pue., Son.)

M. chihuahuensis (A. Gray) Woodson (Chih., Son.)

*M. chrysantha (Greenm.) Woodson (Coah., D.F., Dgo., Gro., Hgo., Jal., Méx., Mich., Mor., Oax., Pue., Qro., Sin., Son., Tlax.)

*M. congesta (Decne.) Woodson (Jal., Oax.)

M. cordifolia (A. Gray) Woodson (B.C., B.C.S., Son.)

${ }^{*}$ M. crassifolia (Standl.) Woodson (Cam., Chis., Q.Roo, Yuc.)

*M. crenata (Vail) Woodson (D.F., Gto., Gro., Hgo., Jal., Méx., Mich., Mor., Oax., S.L.P., Sin., Tlax., Zac.) 
*M. cyclophylla (Standl.) Woodson (Chis., Gro., Jal., Oax., Ver.)

*M. decumbens W. D. Stevens (D.F., Hgo., Méx., Tlax.)

*M. emartinezii W. D. Stevens (Chis.)

*M. fruticosa (Brandegee) Woodson (B.C.S.)

M. fulvida (F. Ballard) W. D. Stevens (Chis.)

M. gentlei (Lundell et Standl.) Woodson (Cam., Chis., Q.Roo, Yuc.)

M. gonoloboides (B. L. Rob. et Greenm.) Woodson (Chis., Jal., Méx., Oax.)

*M. gracilis (Decne.) W. D. Stevens (Oax.)

*M. greggii (Vail) Woodson (Dgo.)

M. guatemalensis (Schum.) Woodson (Chis.)

*M. hastulata (A. Gray) Sundell (B.C.)

*M. inconspicua (Brandegee) Woodson (Oax., Pue.)

${ }^{*} M$. inops Woodson (Chis., Oax.)

*M. ionantha Woodson (Ver.)

*M. johnstonii Shinners (Chih., Son.)

M. lanceolata (Decne.) Woodson (Chis., Oax., Ver.)

*M. lesueurii (Standl.) Woodson (Chih, Son.)

*M. magallanesii E. J. Lott (Jal.)

M. magnifolia (Pittier) Woodson (Chis., Oax., Ver.)

M. medusae Woodson (Chis.)

*M. meyeri Woodson (Tamps.)

M. micrantha L. O. Williams (Cam., Chis.)

M. molinarum L. O. Williams (Chis.)

*M. nigrescens (Schltdl.) Woodson (S.L.P., Ver.)

*M. nummularia (Decne.) Woodson (Dgo., Hgo., Jal., Méx., Mich., Pue.)

*M. oaxacana (Standl.) W. D. Stevens (Oax.)

M. ocellata W. D. Stevens (Mich., Oax.)

M. parviflora (Torr.) Woodson (Coah.)

M. parvifolia (Torr.) Woodson (B.C., B.C.S., Chih., Son.)

M. patalensis (Donn. Sm.) Woodson (Chis.)

*M. pedunculata (Decne.) Woodson (Dgo., Gto., Jal., Méx., Mich.)

*M. petiolaris (A. Gray) Woodson (Chih., Sin., Son.)

M. picturata (Hemsl.) Woodson (Chis)

*M. pilosa (Benth.) Woodson (Dgo., Gto., Hgo., Jal., Mor., Nay., N.L., Oax., Pue., Qro, S.L.P., Sin., Tamps., Ver., Zac.)

M. pringlei (A. Gray) Woodson (B.C., B.C.S., Son.)

M. producta (Torr.) Woodson (Chih., Son.)

*M. prostrata (Willd.) Woodson (Hgo., Pue.)

*M. pueblensis (Brandegee) Woodson (Oax., Pue.) 
*M. purpusii (Brandegee) Woodson (Gro., Oax., Pue.)

M. pusilliflora L. O. Williams (Cam., Chis.)

${ }^{*}$ M. quercetorum (Standl.) W. D. Stevens (Chih., Son.)

M. reticulata (Engelm. ex A. Gray) Woodson (Chih., Coah., Dgo., N.L., Oax., S.L.P., Sin., Tamps.)

*M. rogersii Woodson (N.L.)

M. sagittifolia (A. Gray) Woodson ex Shinners (N.L., Tamps.)

${ }^{*}$ M. schaffneri (Engelm. ex A. Gray) Woodson (Hgo., S.L.P., Zac.)

M. serpens Woodson (N.L.)

*M. stenosepala Lundell (Q.Roo, Yuc.)

M. steyermarkii Woodson (Chis.)

${ }^{*}$ M. suberifera (B. L. Rob.) W. D. Stevens (S.L.P.)

M. sugillata W. D. Stevens (Oax., Qro, Ver.)

M. trachyantha (Greenm.) W. D. Stevens (Chis., Col., Gro., Mich., Mor., Oax., Pue.)

*M. tristiflora (Standl.) Woodson (Chih., Son.)

M. tuerckheimii (Donn. Sm.) Woodson (Chis.)

*M. umbellata (Brandegee) Woodson (B.C.S.)

*M. vailiana Woodson (Chih., Son.)

M. velutina (Schltdl.) Woodson (Cam., Chis., Gto., Hgo., Mor., Oax., Qro., Q.Roo, Sin., Tab., Tamps., Ver., Yuc., Zac.)

M. velutinoides W. D. Stevens (Chis.)

*M. wootonii (Vail) Woodson (Chih.)

Metalepis Griseb.

M. peraffinis (Woodson) Morillo (Chis., Oax., Ver.)

Metastelma R. Br.

*M. angustifolium Turcz. (Chih., D.F., Gto., Hgo., Méx., Mich., N.L., Oax., Pue., Tlax., Ver.)

M. arizonicum A. Gray (Son.)

M. barbigerum Scheele (Chis., Chih., Coah., N.L., Oax., Tamps., Ver., Zac.)

*M. brachymischum W. D. Stevens (Chis.)

${ }^{*}$ M. californicum Benth. (B.C., B.C.S., Col., Son.)

M. chiapense A. Gray (Chis., Oax., Ver.)

*M. cuneatum Brandegee (Sin., Son.)

*M. lanceolatum Schltr. (Chis., D.F., Gro., Hgo., Jal., Mor., Oax., Pue., S.L.P., Yuc.)

*M. latifolium Rose (Chih., Jal., N.L., Sin., Son.)

${ }^{*}$ M. longicoronatum (L. O. Williams) Liede (Chis.)

*M. macropodum Greenm. (Oax., Pue.)

M. mexicanum (Brandegee) Fishbein et R. Levin (Sin., Son.)

*M. minutiflorum Wiggins (Col., Sin., Son.)

M. palmeri S. Watson (Coah., Hgo., N.L., S.L.P., Tamps.)

M. pringlei A. Gray (B.C.S., Chih., Coah., Dgo., N.L., Sin., Son., Tamps.)

*M. pubescens (Greenm.) W. D. Stevens (D.F., Gro., Méx., Mich., Mor., Oax., Pue.) 
*M. schaffneri A. Gray (Gro., Jal., Mor., Oax., S.L.P., Sin., Son.)

M. schlechtendalii Decne. (Cam., Chis., Gro., Mor., Oax., Pue., Q.Roo, Tab., Ver., Yuc.)

M. sepium (Decne.) W. D. Stevens (Hgo., Mich., Oax.)

M. stenomeres (Standl. et Steyerm.) W. D. Stevens (Oax.)

*M. subcordatum Benth. (B.C.S.)

M. thalamosiphon W. D. Stevens (Cam., Chis., Q.Roo, Yuc.)

M. trichophyllum (L. O. Williams) W. D. Stevens (Chis., Oax.)

*M. turneri Liede et Meve (N.L., S.L.P., Tamps.)

M. yucatanense W. D. Stevens (Q.Roo, Yuc.)

Microdactylon Brandegee

*M. cordatum Brandegee (Oax., Pue.)

Orthosia Decne.

O. cynanchoides W. D. Stevens (Chis.)

O. glaberrima (Woodson) W. D. Stevens (Chis.)

O. misera (L. O. Williams) W. D. Stevens (Chis., Ver.)

* O. stipitata W. D. Stevens (Chis.)

Oxypetalum R. Br.

O. cordifolium (Vent.) Schltr. (Cam., Chis., Hgo., Oax., Qro., Q.Roo, S.L.P., Ver., Yuc.)

Pherotrichis Decne.

*P. leptogenia B. L. Rob. (Jal., Mor.)

* P. mixtecana Brandegee (Gro., Oax.)

P. schaffneri A. Gray (Chih., Dgo., S.L.P., Son.)

P. villosa Meisn. (Chis.)

Polystemma Decne.

P. guatemalense (Schltr.) W. D. Stevens (Chis., Col., Gro., Jal., Méx., Mich., Mor., Nay., Oax., Pue., Sin.)

P. viridiflorum Decne. (Chis., Gro., Mor., Oax., Ver.)

Prosthecidiscus Donn. Sm.

P. guatemalensis Donn. Sm. (Chis., Gro., Mich., Nay., Oax., Ver.)

Seutera Rchb.

S. angustifolia (Pers.) Fishbein et W. D. Stevens (Coah., Q.Roo, S.L.P., Tab., Tamps., Ver., Yuc.)

S. palmeri (S. Watson) Fishbein et W. D. Stevens variedad palmeri (B.C.S.)

S. palmeri (S. Watson) Fishbein et W. D. Stevens variedad peninsulare (S.F. Blake) Fishbein et W.D. Stevens (B.C.S.)

Stapelia L.

+S. grandiflora Masson

Tassadia Decne.

T. obovata Decne. (Chis., Oax., Ver.)

Trichosacme Zucc.

T. lanata Zucc. (N.L., S.L.P., Tamps.) 